\title{
Lessons from rodent models in celiac disease
}

\author{
N Korneychuk ${ }^{1,2}$, B Meresse ${ }^{1,2}$ and N Cerf-Bensussan ${ }^{1,2}$
}

Over the past 25 years, studies led in humans have considerably improved our understanding of celiac disease, a complex disease that is generally defined as an autoimmune-like enteropathy induced by dietary gluten in genetically predisposed individuals. Recently, large efforts were also invested in the development of mouse models in order to explore pathogenic hypotheses, and also with the goal to design pretherapeutic models that could be used to test innovative therapies. Yet, modeling this complex multifactorial disease has been a very challenging task. Herein, we review how approaches in rodents have provided insight into celiac disease pathophysiology and also highlight the difficulties met to fully recapitulate the human disease.

\section{INTRODUCTION}

In his seminal "Introduction to the Study of Experimental Medicine" published in 1860, Claude Bernard stressed the necessity for animal experimentation in order to improve the comprehension of human physiology and pathology. He nevertheless anticipated difficulties due to differences in disease susceptibility between humans and animals and suggested to combine animal models to delineate predisposing factors. Interestingly, gluten-sensitive enteropathy is not a disease restricted to genetically predisposed humans but it has also been described in Irish Setters, in Rhesus macaque monkeys, and even in horses (reviewed in ref. 1). Yet, the disease that affects dogs is independent of major histocompatibility complex (MHC) class II genes, the main risk factor for celiac disease (CD) in humans, and the contribution of $\mathrm{MHC}$ is unknown in monkeys and horses. ${ }^{1}$ Moreover, experimental studies in such models are limited for practical and ethical reasons. Therefore, most attempts to build an animal model have been conducted in mice and, recently, in genetically modified mice harboring the risk factors for human CD. Herein, we review how approaches in rodents have provided insight into the complex immunological jigsaw of CD pathophysiology. We also highlight the difficulties met to fully recapitulate human $\mathrm{CD}$ and to design a pertinent model that could be used to test innovative therapies, much needed to alleviate the burden of a life-long gluten-free diet or to treat severe cases that have become refractory to diet.

\section{Pioneering studies in wild-type rodent models}

Typical histological lesions of CD include villous flattening, crypt hyperplasia, and intraepithelial lymphocyte (IEL) infiltration of the proximal intestine. Anne Ferguson was the first in the mid 1970s to point out similarities between intestinal injury in $\mathrm{CD}$ and in mouse models of intestinal allograft rejection or of graft vs. host reaction. Henceforth, she suggested a major role for $\mathrm{T}$ cell-mediated immunity in $\mathrm{CD}$ pathogenesis ${ }^{2,3}$ and examined whether the unusual characteristics of gluten proteins (see Box 1), notably the suggested lectin-like properties of gliadins, influenced the outcome of immune responses. With R Troncone, ${ }^{2}$ she showed that a single feed with gliadins or 1-week-long diet with gluten-containing chow was sufficient to inhibit immunoglobulin $G$ (IgG) antibody responses and systemic delayed hypersensitivity in response to parenteral immunization with gliadin compared with mice maintained on gluten-free diet (see Table 1). The conclusion of this work was therefore that "gliadins are not a particular class of proteins abnormally handled by the gut, and can induce oral tolerance similarly to other dietary proteins such as soluble egg or milk proteins." ${ }^{4}$ As for other dietary proteins, the age at the time of gluten introduction was critical. Whereas pups exposed to gluten after weaning developed oral tolerance, suckling pups fed by mothers on a gluten-containing diet developed systemic immune responses to gluten ${ }^{2}$ (see Table 1). Yet, the lack of intestinal lesions even after systemic immunization and subsequent feeding with gliadin ${ }^{2}$ suggested that mucosal tolerance developed despite the systemic immune response

${ }^{1}$ INSERM UMR1163, Laboratory of Intestinal Immunity, Institut Imagine, Paris, France and ${ }^{2}$ Université Paris Descartes-Sorbonne Paris Cité and Institut Imagine, Paris, France. Correspondence: N Cerf-Bensussan (nadine.cerf-bensussan@inserm.fr) 


\section{Box 1 Gluten}

Gluten is defined as the cohesive and elastic mass left after washing wheat dough with water. Gluten is made of over a 100 distinct proteins comprising gliadin monomers (separated on the basis of their amino acid sequences into $\alpha / \beta, \gamma$, or $\psi$-gliadins) and polymers of glutenins of low or high molecular weights soluble in water alcohol solutions. ${ }^{72}$ Gliadins and glutenins are enriched in proline and glutamine residues and therefore often called prolamines. They form a viscoelastic network that enwraps starch granules and carbonic gas released by fermentation and confer wheat its unique aptitude for bread making. All gluten proteins can be toxic for CD patients but epitopes more often recognized by Tcells from either HLA-DQ2.5 or HLA-DQ8 individuals have been described. ${ }^{73}$ Related proteins rich in proline and glutamine present in rye and barley are also "toxic" for patients. This "toxicity" is ascribed to their content in T-cell epitopes that can activate patients' Tcells. Besides gluten proteins that drive the pathogenic adaptive T-cell response in CD, wheat may contain other proteins, such as lectins or $\alpha$-amylase trypsin inhibitors that were suggested to stimulate inflammatory innate responses ${ }^{74}$ but their exact role in $\mathrm{CD}$ pathogenesis remains unclear.

against gliadin (Table 1). In contrast, Stepankova et al. ${ }^{5}$ observed villous blunting, crypt hyperplasia, and expansion of $\mathrm{CD}^{+}{ }^{+} \mathrm{TCR} \alpha \beta^{+}$IELs in young germ-free rats administered gliadin intragastrically from birth until day 63. An enteropathy was also induced in conventionally raised neonate rats when combining gluten gavage, feeding with artificial milk, and intraperitoneal injection of interferon- $\gamma(\operatorname{IFN} \gamma)^{6}$ (Table 1). Overall, these data indicate that dietary gluten generally leads, alike other dietary proteins, to systemic and mucosal tolerance. At a very young age, factors such as amount of gluten, components present in breast milk, inflammatory cytokines produced in response to infections, and perhaps the microbiota might influence the development of tolerance. Although these studies did not demonstrate any distinctive immunogenic features of gluten compared with other dietary proteins, it must be stressed that gluten proteins, because of their high content in proline, are not easily digested by intestinal and brush border enzymes, resulting in the release in the duodenal lumen of large immunogenic peptides that may promote immune responses in genetically predisposed individuals. ${ }^{7}$

\section{Lessons from gluten-induced intestinal immune responses in immunodeficient mice}

Over the past 20 years, human studies have highlighted the central role of gluten-specific intestinal $\mathrm{CD}^{+}{ }^{+} \mathrm{T}$ cells in CD pathogenesis (reviewed in refs. 8 and 9). Henceforth, Freitag et al. ${ }^{10,11}$ developed a model of gluten-induced small intestinal enteropathy adapted from the transfer model of colitis in immunodeficient mice (Table 1). C57BL/6 mice were immunized twice with gliadin (or as a control with ovalbumin) in complete Freund's adjuvant to activate gluten-specific $\mathrm{CD}^{+}{ }^{+} \mathrm{T}$ cells. Spleen memory CD $4{ }^{+} \mathrm{CD} 45 \mathrm{RB}^{\text {low }} \mathrm{CD} 25^{-} \mathrm{T}$ cells deprived of regulatory $\mathrm{T}$ cells were then sorted and intraperitoneally injected into $\mathrm{T}$ and $\mathrm{B}$ cell-deficient $\mathrm{RAG1}{ }^{-/-}$or into $\mathrm{T}$ cell-deficient nude mice, and recipients were fed with gluten-rich or gluten-free diet for 8.5 weeks $^{10,11}$ (Table 1). Injection of gluten-sensitized $\mathrm{T}$ cells into gluten-fed nude mice induced serum anti-gliadin IgG and IgA antibodies, but no significant enteropathy. ${ }^{10}$ In contrast, RAG1 ${ }^{-1}$ recipients injected with gluten-sensitized $\mathrm{CD}^{+} \mathrm{T}$ cells developed duodenal epithelial lesions. ${ }^{10,11}$ In mice fed on gluten-free diet, lesions remained mild and subsided after oral treatment with antibiotics, in agreement with the known role of the microbiota in this model. ${ }^{11}$ Epithelial lesions were however considerably aggravated by a gluten-containing diet that induced significant weight loss and severe duodenitis with enhanced levels of IFN $\gamma$ and interleukin (IL)-17 duodenal transcripts. ${ }^{10,11}$ The authors therefore concluded that $\mathrm{CD} 4{ }^{+}$ T-cell immunity against gluten could, in combination with signals from the microbiota, trigger a severe enteropathy in lymphopenic mice. ${ }^{10,11}$ This study raises several questions. First, what is the mechanism of epithelial damage? Is it only dependent on $\mathrm{CD}^{+}{ }^{+} \mathrm{T}$ cells? In CD, epithelial damage is likely mediated by cytotoxic CD8 ${ }^{+}$T-IELs (see below). RAG1 ${ }^{-1-}$ mice are deficient in $\mathrm{CD} 8{ }^{+} \mathrm{T}$-IELs but they possess substantial numbers of intraepithelial innate-like cells, some of which display cytotoxicity and could perhaps participate in tissue damage. ${ }^{12}$ Second, what is the role of FoxP3 ${ }^{+}$regulatory T cells (Tregs)? Tregs are present in normal or even increased numbers in CD mucosa. ${ }^{13-15}$ Tregs can prevent the development of inflammation in the original transfer model of colitis. ${ }^{16}$ It would thus be interesting to compare the enteropathy in mice injected with sensitized $\mathrm{T}$ cells not depleted in CD $25^{+}$Tregs. Third, why an enteropathy developed in $\mathrm{Rag} 1^{-/-}$mice but not in nude mice injected with gliadin-sensitized T cells? In contrast with Rag1 ${ }^{-1-}$ mice, nude mice developed a strong serum antigliadin IgG and IgA response ${ }^{10}$ (Table 1). Intestinal secretory IgAs (SIgAs) were not studied but their release might have prevented gluten absorption, and subsequent $\mathrm{T}$-cell activation and enteropathy. This hypothesis is supported by studies showing that in humans, but not in mice, SIgA can play a Trojan horse role and facilitate intestinal permeability to gluten (see below).

\section{Lessons from studies in humanized HLA-DQ2.5/8 transgenic mice}

Studies initiated over 20 years ago have shown that two MHC class II molecules, HLA-DQ2.5 and HLA-DQ8, are present in 90\% and $10 \%$ of patients, respectively, and confer the main genetic risk factor (reviewed in refs. 8 and 9). The two molecules were shown to have peptide pockets that can selectively accommodate prolineand glutamine-rich gluten peptides. HLA-DQ2.5 and HLA-DQ8 can thereby facilitate the activation of gluten-specific $\mathrm{CD} 4{ }^{+} \mathrm{T}$ cells. ${ }^{8,9}$ Following the success of a humanized rat model to demonstrate the instrumental role of HLA-B27 in driving arthritis and gut inflammation, ${ }^{17}$ humanized mice were produced to confirm the role of human leukocyte antigen (HLA) class II genes in inflammatory pathologies and notably in CD.

Transgenic mice harboring HLA-DQ8 $\alpha$ and $\beta$ genes and, as a control HLA-DQ6 $\alpha$ and $\beta$ genes, were obtained by David 


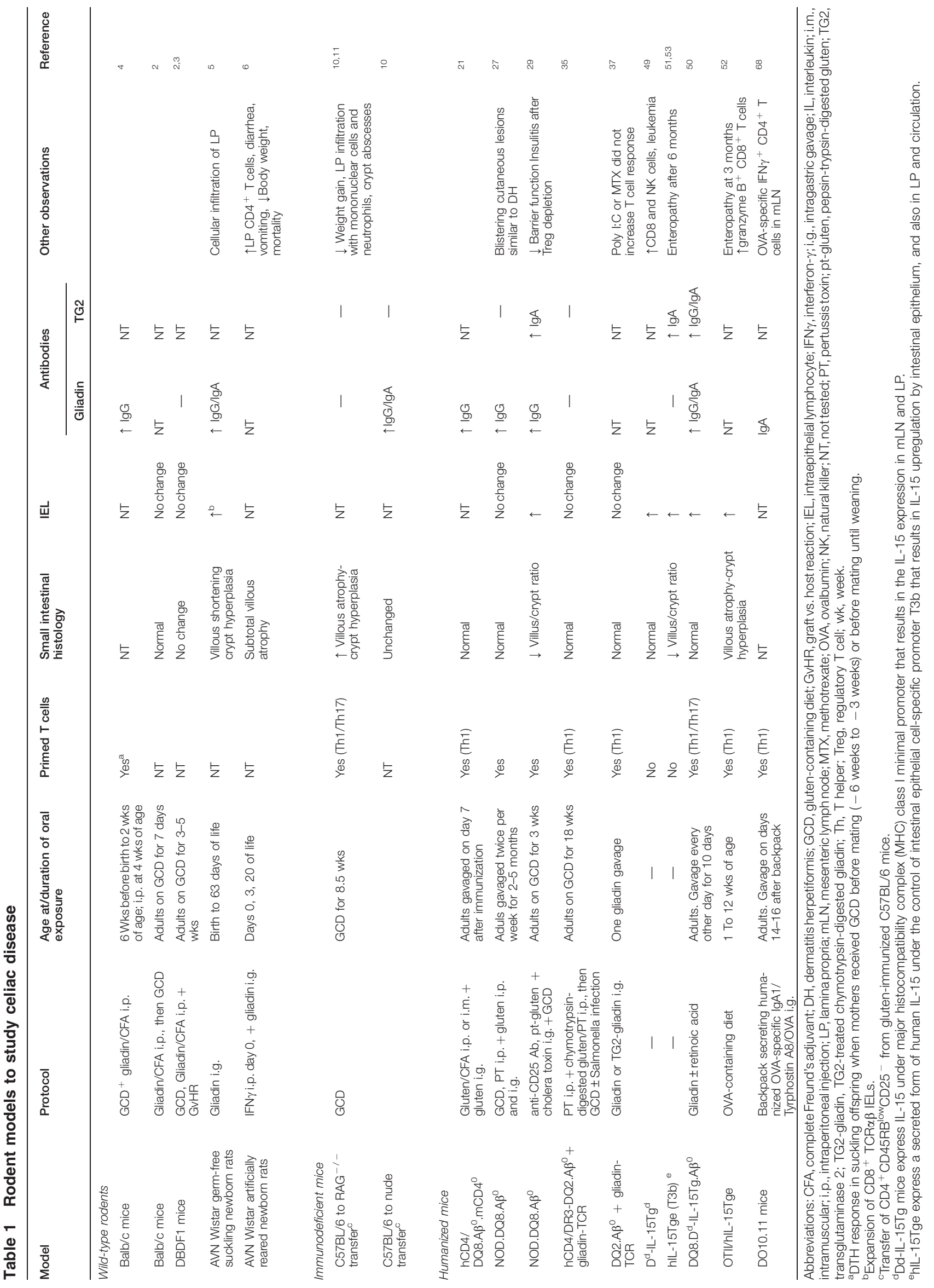


et al. ${ }^{18,19}$ In order to avoid any competition between the human DQ and the endogenous MHC class II molecules, the human genes were introduced in $\mathrm{H} 2-\mathrm{A} \beta^{\circ} \mathrm{C} 57 \mathrm{BL} / 6$ mice $\left(\mathrm{A} \beta^{\circ}\right)$ that cannot express endogenous mouse $\mathrm{MHC}$ class II and have almost no $\mathrm{CD}^{+}{ }^{+} \mathrm{T}$ cells $\left(3-7 \%\right.$ in spleen) ${ }^{20}$ HLA-DQ molecules were observed on $15-30 \%$ of peripheral mouse mononuclear cells. Of the T cells, $10-13 \%$ were $\mathrm{CD}^{+}$and displayed a diverse $\mathrm{T}$-cell repertoire, indicating that $\mathrm{CD} 4^{+}$ T-cell selection was partially restored by the human DQ transgenes (reviewed in ref. 19). When immunized with heterologous collagen II, DQ8.A $\beta^{\circ}$ mice developed a much more severe arthritis than DQ6 DQ6.A $\beta^{\circ}$ mice. ${ }^{18,19}$ This result was in accordance with the notion that DQ8 but not DQ6 predisposes to rheumatoid arthritis and supported the view that $\mathrm{DQ} 8 . \mathrm{A} \beta^{\circ}$ mice might be a pertinent model for in vivo analysis of DQ8-restricted human $\mathrm{CD}^{+}{ }^{+} \mathrm{T}$ cell responses. DQ8.A $\beta^{\circ}$ mice were therefore used by Murray and colleagues ${ }^{21}$ to analyze $\mathrm{CD}^{+}{ }^{+}$T-cell responses to gluten. To further facilitate the generation of DQ-restricted $\mathrm{CD} 4{ }^{+} \mathrm{T}$ cells, the latter mice were crossed with mice harboring a human CD4 transgene but lacking the mouse endogenous CD4 gene ${ }^{21,22}$ (Table 1). HCD4/ DQ8 mice immunized in the footpad with crude gluten and complete Freund's adjuvant developed much stronger proliferative T-cell responses in the draining lymph nodes than hCD4/DQ6 mice. Proliferation was inhibited by a DQ antibody and could be demonstrated for a spectrum of gluten peptides, including gliadin peptide 206-217, an immunodominant peptide in HLA-DQ8 celiac patients ${ }^{21,23}$ (see Box 1). Importantly, the T-cell response in HCD4/DQ8 mice was stronger against a modified peptide 206-217, in which glutamine residues 208 and 216 were replaced by glutamic acid to mimic its post-translational modification by transglutaminase 2 (TG2). ${ }^{21,24}$ Indeed, TG2, the target of CD-specific IgA autoantibodies, can catalyze the deamidation of gluten peptides, and thereby create negative charges that enhance their binding to HLA-DQ2 or-DQ8 (reviewed in ref. 8). Overall, these data confirmed that HLA-DQ8 promotes gluten recognition and that, in vivo, as already observed in vitro, ${ }^{23,24}$ deamidation is not absolutely required for but enhances HLA-DQ8 gluten presentation to CD4 ${ }^{+} \mathrm{T}$ cells. The role of DQ8 in the onset of a pathogenic intestinal $\mathrm{CD}^{+}{ }^{+} \mathrm{T}$-cell response to gluten was next tested using a single gavage of gluten 7 days after subcutaneous immunization. On day 14, hCD4/DQ8 mice developed much higher titers of anti-gliadin IgG than hCD4/DQ6 mice, but they displayed neither IgA against gluten and TG2 nor intestinal pathology (Table 1). Interestingly, intestinal and spleen cells in vitro restimulated with gluten produced IFN $\gamma$, IL-2, and IL-6, as well as significant amounts of regulatory cytokines (IL-10 and transforming growth factor- $\beta$ ), and no or only small differences were found between hCD4/DQ8 and hCD4/DQ6 mice. ${ }^{21}$ Therefore, even after systemic immunization, a short oral exposure to gluten was not sufficient to trigger a pathological gut immune response in hCD4/DQ8 mice (Table 1). This result, which contrasts with those obtained in immunodeficient mice (see above), led the authors to consider not only the duration of the diet but also the role of additional genetic or environmental factors that may impair immunoregulation.

As $C D$ is strongly associated with type 1 diabetes ${ }^{25}$ and shares genetic predisposing factors, including DQ8 and a large spectrum of genetic variants revealed by genome-wide scans, ${ }^{26}$ HLA-DQ8.A $\beta^{\circ}$ mice were backcrossed on the autoimmuneprone NOD background, and intraperitoneally immunized with gluten and a distinct adjuvant, pertussis toxin ${ }^{27}$ (Table 1). Neither intestinal lesions nor diabetes were observed but, strikingly, within 2 to 5 months on a gluten-containing diet, a substantial fraction of NOD A $\beta^{\circ}$ DQ8 mice developed blistering cutaneous lesions reminiscent of dermatitis herpetiformis (DH), a gluten-induced cutaneous disease associated with $\mathrm{CD}^{27}$ (Table 1). As in DH, blisters contained neutrophils and monocytes, were associated with perilesional dermal IgA deposits, and disappeared after 3 weeks of gluten-free diet or even faster if the diet was associated with topical application of steroids. In this model and as suspected in $\mathrm{DH}$, chronic activation of DQ8-restricted $\mathrm{CD} 4{ }^{+} \mathrm{T}$ cells by dietary gluten might stimulate the production of IgA able to deposit in dermis and to attract elastase-secreting neutrophils that trigger blistering. Yet, and in contrast with $\mathrm{DH}$, mice displayed only serum IgG against gliadin, and no IgA against either TG2 or its epidermal homolog TG3, the suspected autoantigen in $\mathrm{DH},{ }^{28}$ could be detected (Table 1).

A different outcome was observed by Verdu and colleagues, ${ }^{29}$ who used the same NOD Ab ${ }^{\circ}$ DQ8 mice but a different protocol of immunization (Table 1). In this second study, mice were gavaged once a week for 3 weeks with pepsin-trypsin digests of gliadin and cholera toxin, a strong adjuvant of gut immune responses that also enhances intestinal permeability (Table 1). Mice did not develop cutaneous lesions but displayed increased numbers of IELs, increased epithelial conductance (reflecting increasing permeability to ions), and a mild enteropathy with elongated crypts that was not observed in control mice immunized with bovine serum albumin ${ }^{29}$ (Table 1). Out of 11 gliadin-sensitized mice, 3 also had moderately increased levels of serum anti-TG2 IgA (Table 1). Partial depletion of $\mathrm{CD} 25^{+}$Tregs before oral immunization did not aggravate intestinal lesions but resulted in insulitis, although without overt diabetes ${ }^{29}$ (Table 1). Overall, these data show that recognition of gluten by HLA-DQ8-restricted CD $4^{+}$ $\mathrm{T}$ cells is not sufficient to trigger gluten-associated pathology, but that additional genetic and/or environmental factors that influence the nature and site of the immune response are involved. The onset of insulitis upon oral exposure to gluten has been reported in other animal models of type 1 diabetes ${ }^{30,31}$ and is in line with the clinical association between diabetes and CD. Of note, both duodenum and pancreas are drained into duodenopancreatic lymph nodes in which sensitization against dietary proteins might take place. ${ }^{32}$ Moreover, pancreatic and intestinal capillaries express MadCAM (mucosal addressin cell adhesion molecule-1) and can both recruit lymphocytes primed in gut-associated lymphoid tissue. ${ }^{33,34}$ It is however unclear whether insulitis may be promoted by gliadin-sensitized $\mathrm{T}$ cells. Recent work rather suggests that gluten may influence the 
composition of microbiota and as a consequence the expansion of Tregs. ${ }^{31}$

If HLA-DQ8 is the main MHC class II haplotype predisposing to type 1 diabetes, it is only present in $10 \%$ of $\mathrm{CD}$ patients, with $90 \%$ of the patients rather expressing HLADQ2.5. ${ }^{8,9}$ The role of HLA-DQ2.5 was therefore also addressed in humanized transgenic models. Mice that expressed a large 320-kb region of the HLA-DR3-DQ2.5 MHC and human CD4 but lacked mouse class II molecules were generated by McCluskey and colleagues ${ }^{35}$ (Table 1). In hCD4.DR3-DQ2.A $\beta^{\circ}$ mice, the HLA transgenes were detected on dendritic cells, B cells, and on a fraction of macrophages, whereas $18 \%$ of splenic lymphocytes displayed the human CD4 molecule and diverse T-cell receptor- $\beta$ (TCR $\beta$ ) chains. ${ }^{35}$ In humans, efficient HLA-DQ2.5 presentation of gliadin peptides to $\mathrm{CD}^{+} \mathrm{T}$ cells requires their deamidation by TG2. ${ }^{8}$ Accordingly, $\mathrm{T}$ cells from hCD4.DR3-DQ2.A $\beta^{\circ}$ mice immunized with a deamidated version of a DQ2-restricted $\alpha$-gliadin peptide (57-73 Q65E) specifically responded to in vitro recall with the same peptide, whereas the non-deamidated peptide triggered only a very weak T-cell response. ${ }^{35}$ Disappointingly however, feeding hCD4.DR3-DQ2.A $\beta^{\circ}$ mice with a glutencontaining diet failed to induce any intestinal pathology (Table 1). Several immunization protocols were used to sensitize mice before gluten feeding. Subcutaneous immunization in complete Freund's adjuvant had no effect (Table 1). Only 2 of 14 transgenic mice immunized intraperitoneally with gluten and pertussis toxin developed serum IgA against TG2, one of which also had increased numbers of IELs and elongated crypts but normal villi. Crossing hCD4.DR3-DQ2.A $\beta^{\circ}$ mice on a NOD background did not accentuate the gluten-induced phenotype. Moreover, infecting mice with Salmonella typhimurium to cause tissue damage and to putatively activate TG2 did not enhance the severity or incidence of TG2 antibodies or of enteropathy. ${ }^{35}$ In order to further increase the number of gluten-specific $\mathrm{T}$ cells, the authors generated mice that also expressed a gliadin-specific TCR (Table 1). HCD4.DR3-DQ2.A $\beta^{\circ}$ mice were immunized with $\alpha$-gliadin $57-73$ Q65E and their T cells were used to derive specific hybridomas. ${ }^{35}$ The $\alpha$ and $\beta$ TCR genes of one hybridoma specific of 57-73 Q65E were used to produce a hCD4.DR3-DQ2.A $\beta^{\circ}$ mouse line with a transgenic gliadinspecific TCR. In the latter mice, $97 \%$ of peripheral $\mathrm{CD} 4^{+} \mathrm{T}$ cells expressed the transgene-encoded TCR, demonstrating their efficient intrathymic selection. These $\mathrm{T}$ cells proliferated in response to $\alpha$-gliadin $57-73$ either in vitro or in vivo after transfer into immunized hCD4.DR3-DQ2.A $\beta^{\circ}$ mice. Significant proliferation of transferred $\mathrm{T}$ cells was also observed in Peyer's patches and mesenteric lymph nodes (mLNs) of mice gavaged with the 57-73 Q65E peptide for 3 days. Moreover, $\mathrm{CD}^{+}{ }^{+} \mathrm{T}$ cells of transgenic mice displayed a TH1 profile upon in vitro restimulation with $57-73$ Q65E. ${ }^{35}$ Despite such convincing evidence that gliadin-specific $\mathrm{CD} 4{ }^{+} \mathrm{T}$ cells were functional and activated in vivo even after mucosal delivery of the immunogenic peptide, none of those mice developed TG2specific IgA antibodies or discernible signs of intestinal pathology after gluten gavage, even if coadministered with pertussis toxin or coinfected with S. typhimurium ${ }^{35}$ (Table 1). Overall, these data support the view that gliadin-specific $\mathrm{CD} 4{ }^{+} \mathrm{T}$ cells are not sufficient to induce the development of $\mathrm{CD}$. It is possible that, because of the lack of activation of TG2 at steady state, only a small number of the latter cells might have been activated. Yet, the frequency of gliadin-specific $\mathrm{CD} 4^{+} \mathrm{T}$ cells in the intestine of untreated CD patients, estimated by limiting dilutions or using HLADQ2.5 tetramers, is between 0.2 and $2 \%,{ }^{36}$ and thus largely below the frequency of such cells in the mouse model. A more likely hypothesis is that tolerance to gluten develops in gluten-fed humanized mice as it does in wild-type mice (see above).

This hypothesis is supported by results obtained by Samson and co-workers ${ }^{37}$ (Table 1). These authors used a distinct HLADQ2.5 model transgenic mice in which the MHC transgene was a smaller $68 \mathrm{~kb}$ fragment encompassing $\mathrm{DQA}^{*} 0501$ and DQB $1^{*} 0201$ genes including promoters and regulatory elements, ${ }^{37}$ whereas the transgenic TCR was made of the variable domains of the $\alpha$ and $\beta$ chains of a human $\gamma 1$-gliadinspecific $\mathrm{T}$-cell clone combined with constant regions and regulatory elements of mouse origin. ${ }^{38}$ As in the first model, spleen cells from HLA-DQ2.gliadin-TCR.A $\beta^{\circ}$ double transgenic mice contained a substantial number of $\mathrm{CD} 4^{+}$transgenic cells that responded to deamidated but not to native gliadin and produced a spectrum of either proinflammatory (IL-2, IFN $\gamma$, IL-17, IL-21, and IL-4) or regulatory (IL-10) cytokines ${ }^{37}$ (Table 1). Interestingly, when transgenic T cells from HLADQ2.gliadin-TCR.A $\beta^{\circ}$ mice were transferred into HLADQ2.5.A $\beta^{\circ}$ mice gavaged with deamidated gliadin, they underwent strong proliferation in spleen, but strikingly, the proliferating cells displayed regulatory function (Table 1). Indeed, an additional transfer of these isolated cells suppressed skin hypersensitivity induced in HLA-DQ2.gliadin-TCR.A $\beta^{\circ}$ double transgenic mice by systemic immunization and subcutaneous challenge with TG2-treated gliadin. As the regulatory TCR transgenic $\mathrm{T}$ cells did not express FoxP3 (forkhead box P3) but produced large amounts of IL-10, they were considered type 1 Tregs. ${ }^{37}$ The lack of induction of Foxp $3^{+}$Tregs and the preferential localization of the proliferating Tregs in spleen but not in $\mathrm{mLN}$ was surprising given previous observations in mice fed with ovalbumin (OVA), a model in which oral tolerance depends on the induction of Foxp3 ${ }^{+}$Tregs in mLN. ${ }^{37,39}$ Nevertheless, distinct mechanisms might be involved in the establishment of tolerance to dietary proteins.

In conclusion, analysis of humanized mouse models confirms that HLA-DQ2.5 as well as HLA-DQ8 molecules facilitate the in vivo $\mathrm{CD} 4{ }^{+} \mathrm{T}$-cell responses against deamidated gluten peptides. Yet, the quantitative increase in gliadinspecific $\mathrm{CD} 4^{+} \mathrm{T}$ cells is not sufficient to switch off tolerance induced by oral gluten. Additional mechanisms are necessary to break tolerance, stimulate the expansion and activation of cytotoxic $\mathrm{CD} 8{ }^{+}$IELs, and ultimately induce intestinal tissue damage and pathology. 


\section{Lessons from IL-15 transgenic mice}

We and others have suggested that IL-15 plays an important role in CD (reviewed in refs. 9 and 40). IL-15 can be produced by almost any cell type, but is secreted in very small amounts and remains bound at the cell surface to the ubiquitous $\alpha$ chain of the IL-15R receptor. This complex can activate nearby lymphocytes, notably CD8 ${ }^{+} \mathrm{T}$ cells, natural killer $(\mathrm{NK})$ cells, and IELs that express the signaling part of IL-15 receptor that consists of the IL-2/IL-15R $\beta$ and $\gamma c$ chains. ${ }^{9,40}$ Both IL-15 (see refs. ${ }^{41,42}$ ) and IL-15R $\alpha^{43}$ are upregulated in the intestinal epithelium and lamina propria of CD patients, and several studies in human CD have highlighted the role of IL-15 in the expansion, cytotoxic activation, and also malignant transformation of IELs. ${ }^{41,42,44-46}$ Moreover, IL-15 interferes with immunoregulatory mechanisms. ${ }^{13,14,47,48}$ The role of IL-15 was analyzed in mice expressing an IL-15 transgene with the leader peptide of IL-2 in order to enhance secretion. In a first model, the IL-15 gene was of mouse origin and placed under the control of the ubiquitously active MHC class $\mathrm{I} H 2-\mathrm{D}^{\mathrm{d}}$ promoter $\left(\mathrm{H} 2-\mathrm{D}^{\mathrm{d}} \text {-IL-15Tg mice }\right)^{49}$ (Table 1). H2-D ${ }^{\mathrm{d}}$-IL-15Tg mice showed a massive expansion of $\mathrm{NK}$ and $\mathrm{CD} 8{ }^{+}$memory $\mathrm{T}$ cells in lymphoid and nonlymphoid organs, and $\sim 50 \%$ developed leukemia consisting of clonal $\mathrm{CD}^{+} \mathrm{T}$ cells expressing NK receptors ${ }^{49}$ (Table 1). The latter observation provides a rationale for the increased risk of $\mathrm{CD}$ patients to develop IEL-derived lymphomas that, for many years, remain dependent on IL-15. ${ }^{42,46}$ In this first model, IL-15 was produced in lamina propria but not in epithelium and no increase in numbers of intestinal CD8 ${ }^{+}$T cells or IELs was reported. ${ }^{50}$ In a second model, an IL-15 transgene of human origin was placed under the control of the enterocyte-specific promoter T3b (hIL15Tge mice $)^{51}$ (Table 1). Homozygous hIL-15Tge mice died rapidly after birth likely from leukemias, but heterozygous mice developed a slowly progressing expansion of intestinal CD8 ${ }^{+}$ $\mathrm{TCR} \alpha \beta \mathrm{T}$ cells and, after several months, a chronic severe enteropathy partially mimicking the pathology was observed in $\mathrm{CD}^{51}$ (Table 1). In hIL-15Tge mice, intestinal $\mathrm{CD}^{+} \mathrm{T}$ cells produced increased amounts of IFN $\gamma$ and tumor necrosis factor- $\alpha$; a small fraction upregulated NK receptors (NK1.1 and NKG2D). ${ }^{51,52}$ Progression toward enteropathy was also associated with the accumulation of granzyme $\mathrm{B}^{+} \mathrm{CD}^{+} \mathrm{T}$ cells. $^{52}$ Moreover, hIL-15Tge mice displayed increased numbers of lamina propria plasma cells and serum IgA against $\mathrm{TG} 2^{53}$ (Table 1). In contrast with $\mathrm{CD}$, in which $\mathrm{CD} 8{ }^{+} \mathrm{T}$ cells expand mainly in epithelium, $\mathrm{CD}^{+}{ }^{+} \mathrm{T}$ cells expanded also in lamina propria because of diffusion of the secreted transgenic IL-15. Treatment of hIL-15Tge mice with antibodies blocking IL-15 or the $\beta$ chain of its receptor or with a drug inhibiting Janus kinase 3, a key molecule in the anti-apoptotic signaling cascade triggered by IL-15, abolished the CD8 infiltration and allowed epithelial recovery. ${ }^{46,54,55}$ These results provide a rationale for the use of drugs blocking IL-15 in the rare but severe cases of CD that become refractory to gluten-free diet, notably when resistance is characterized by the development of an IL-15-dependent intraepithelial lymphoma (reviewed in ref. 56). These results also highlight the capacity of intestinal cytotoxic $\mathrm{CD}^{+}{ }^{+} \mathrm{T}$ cells to induce tissue damage. Yet, they do not explain the indispensable role of $\mathrm{CD} 4{ }^{+} \mathrm{T}$ cells specific for gluten in triggering CD. The interactions between IL-15 and intestinal $\mathrm{CD}^{+}{ }^{+} \mathrm{T}$ cells were examined in two other models.

\section{Models combining IL15-overexpression and activation of intestinal $\mathrm{CD}^{+}{ }^{+} \mathrm{T}$ cells by dietary antigens}

In a first model, Jabri and colleagues ${ }^{50}$ crossed humanized HLA-DQ8.A $\beta^{\circ}$ mice $^{20}$ with H2-D ${ }^{\mathrm{d}}$-IL-15Tg (Table 1). HLADQ8. ${ }^{\mathrm{d}}$-IL-15Tg mice fed with gliadin every other day for 10 days did not develop any intestinal damage but displayed increased levels of serum anti-gliadin and anti-TG2 antibodies, increased frequency of IFN $\gamma$-producing $\mathrm{T}$ cells in $\mathrm{mLN}$ and lamina propria, and increased IEL numbers compared with mice on control diet ${ }^{50}$ (Table 1). These changes were suggested to mimic latent $\mathrm{CD}$ that can precede the onset of villous atrophy and overt $\mathrm{CD}$ (reviewed in ref. 9). The short exposure of mice to gluten as well as the lack of expression of IL-15 in the gut epithelium might explain why mice did not develop epithelial damage.

In order to investigate whether and how intestinal CD4 ${ }^{+} \mathrm{T}$ cells and epithelium-derived IL-15 might cooperate to generate tissue damage, we designed yet another model. In humanized DQ2 mice, the generation of gluten-specific T cells is hampered by the low level of TG2 expression necessary for deamidation of dietary gluten peptides and their efficient presentation to $\mathrm{T}$ cells. To circumvent this problem, we used OTII mice, which possess $\mathrm{CD} 4{ }^{+}$T cells expressing a transgenic TCR specific for a peptide of OVA, recognized without post-translational modification. ${ }^{57}$ OTII mice were crossed with hIL-15Tge mice that, in contrast to $\mathrm{H} 2-\mathrm{D}^{\mathrm{d}}$-IL15Tg mice, overexpress IL-15 in the small intestinal epithelium $^{51,52}$ (Table 1). The OTII ${ }^{+/-}$B6 and OTII $^{+/-}$hIL-15Tge $^{+/-}$offspring were exposed to OVAcontaining or control diets $^{52}$ (Table 1). As hIL-15 Tge $^{+/-}$mice develop a spontaneous enteropathy after 4-5 months, we decided to limit our analysis to the first 3 months of life (Table 1). Giving OVA diet for 1 month after weaning was not sufficient to induce weight loss and significant epithelial changes (E. Ramiro, unpublished results), suggesting that either exposure to the antigen was too short or tolerance mechanisms were difficult to break after weaning. Therefore, OVA diet was introduced in pregnant mothers 8 days before delivery, and pursued during weaning in mothers and after weaning in the offspring $^{52}$ (Table 1). OTII ${ }^{+/}$B6 control mice chronically exposed to OVA showed normal growth and intestinal architecture. Suggesting active tolerance, the frequency of OVA-specific FoxP3 was increased in the intestinal lamina propria, whereas OVA-specific effector $\mathrm{CD}^{+} \mathrm{T}$ cells were depleted in and outside the gut. ${ }^{52}$ These data support the notion that increasing the numbers of $\mathrm{CD}^{+}{ }^{+} \mathrm{T}$ cells specific for a dietary antigen is not sufficient to prevent the establishment of tolerance. ${ }^{58}$ In contrast, a substantial fraction of OTII ${ }^{+/-}$hIL- $^{-}$ 15Tge ${ }^{+}$mice exposed to OVA and notably almost all females developed growth retardation, villous atrophy. and a conspicuous expansion of granzyme B-expressing $\mathrm{CD} 8{ }^{+} \mathrm{T}$ cells in the duodenal mucosa ${ }^{52}$ (Table 1). Strikingly, these changes 
occurred despite the expansion of lamina propria OVA-specific Foxp $3^{+}$Tregs. In contrast to previous observations in HLADQ8. ${ }^{\mathrm{d}}$-IL-15Tg mice, ${ }^{50}$ IL-15 did not prevent in vivo conversion of naive $\mathrm{CD} 4{ }^{+} \mathrm{T}$ cells from OTII $\mathrm{RAG}^{-1-}$ mice into FoxP3 ${ }^{+}$Tregs. $^{52}$ In agreement with human data, ${ }^{13,14,47,48}$ we observed that, in the presence of IL-15, effector T cells, notably $\mathrm{CD} 8{ }^{+} \mathrm{T}$ cells, failed to respond to FoxP3 ${ }^{+}$Tregs and did not restrain their production of IFN $\gamma$ or granzyme $\mathrm{B}$ (Figure 1). Consistent with impaired control of effector T-cell responses by FoxP3 ${ }^{+}$Tregs, hIL-15Tge ${ }^{+/-}$mice displayed increased frequency of IFN $\gamma$-secreting $\mathrm{CD} 4^{+}$or $\mathrm{CD} 8^{+}$cells in and outside the gut. However, this change was observed in all hIL-15Tge mice independently of the diet and was not always associated with intestinal lesions. In contrast and as observed in $\mathrm{CD}$, intestinal damage correlated with an increase in granzyme $\mathrm{B}^{+} \mathrm{CD}^{+} \mathrm{T}$ cells and depended both on IL-15 and activation of specific $\mathrm{CD} 4^{+} \mathrm{T}$ cells by a dietary antigen. ${ }^{52}$

In $\mathrm{CD}$, it remains unclear whether intestinal $\mathrm{CD} 8^{+}$ $\mathrm{T}$ cells can recognize gluten peptides (reviewed in ref. 9). Strikingly, intestinal CD8 ${ }^{+}$T cells of OTII ${ }^{+/-}$hIL-15Tge $^{+}$ mice exposed to OVA and developing intestinal damage showed no specificity for OVA. ${ }^{52}$ We therefore examined how OVA-specific $\mathrm{CD}^{+}{ }^{+} \mathrm{T}$ cells might promote the generation of noncognate cytotoxic $\mathrm{T}$ cells in hIL-15Tge mice. We observed that, in the presence of IL-15, OVA-specific $\mathrm{CD} 4^{+} \mathrm{T}$ cells stimulated by their specific peptide provided help to noncognate effector/memory $\mathrm{CD} 8^{+} \mathrm{T}$ cells, resulting in their proliferation and upregulation of granzyme B and NKG2D (Figure 1). Moreover, $\mathrm{CD} 4^{+} \mathrm{T}$-cell help depended exclusively on a soluble factor and could be recapitulated in vitro as well as in vivo by IL-2 (Figure 1). Injection of IL-2 into hIL-15Tge mice (but not in $\mathrm{B} 6$ mice) resulted in expansion of granzyme $\mathrm{B}^{+}$ intestinal $\mathrm{CD}^{+} \mathrm{T}$ cells and in reduction of the villus/crypt ratio. Importantly, only effector/memory but not naive $\mathrm{CD} 8^{+}$ $\mathrm{T}$ cells responded to the complementary signals provided by IL15 and OVA-specific CD4 ${ }^{+}$T cells. ${ }^{52}$ We suggest that such cooperation takes place in the intestinal mucosa of $\mathrm{CD}$ patients between gluten-specific DQ-restricted $\mathrm{CD}^{+}{ }^{+} \mathrm{T}$ cells, epithelium-derived IL-15, and $\mathrm{CD} 8^{+}$T-IELs that might then progressively expand and acquire granzyme $\mathrm{B}$ whatever their specificity (Figure 1). Accumulation of cytotoxic IEL might then be sustained by the anti-apoptotic signals delivered by IL15 (Figure 1). In human $\mathrm{CD}$, gluten-specific $\mathrm{CD} 4^{+} \mathrm{T}$ cells might also synergize with IL-15 via their production of IL-21, a cytokine not detected in the mouse model..$^{59-61}$ Yet, if the cytotoxic $\mathrm{CD} 8^{+} \mathrm{T}$ cells that expand in $\mathrm{CD}$ are not specific for the dietary gluten, what are the mechanism(s) that trigger IEL degranulation and tissue damage? TCR triggering by cognate antigens of microbial or of self-origin is possible. As previously suggested by ex vivo studies in human $\mathrm{CD},{ }^{44,45,62}$ degranulation might also be triggered via NK receptors and notably via NKG2D constitutively expressed by human $\mathrm{CD} 8^{+} \mathrm{T}$ cells. This receptor can interact with its ligand MICA upregulated in the epithelium of CD patients. ${ }^{44}$ Rae-1, the mouse homolog of MICA ${ }^{63}$ is expressed in the intestine of both control and hIL15Tge mice. ${ }^{52,55}$ Yet, NKG2D is not constitutively expressed by mouse $\mathrm{CD} 8{ }^{+} \mathrm{T}$ cells and was only moderately upregulated on intestinal $\mathrm{CD}^{+}{ }^{+} \mathrm{T}$ cells of $\mathrm{OTII}^{+/-}$hIL-15Tge $^{+/-}$mice exposed to OVA. ${ }^{52}$ The difference in NKG2D expression between humans and mice may explain the increased resistance of mice to the induction of tissue damage by dietary antigens. Conversely, the noncognate help provided by $\mathrm{CD} 4{ }^{+} \mathrm{T}$ cells may not only activate effector/memory $\mathrm{CD} 8^{+} \mathrm{T}$ cells but also $\mathrm{NKG}_{2} \mathrm{D}^{+}$NK cells or NK-like innate lymphoid cells. Such cells are numerous in $\mathrm{Rag}^{-1-}$ mice and may contribute to the epithelial lesions induced by the transfer of gluten-specific $\mathrm{CD}^{+} \mathrm{T}$ cells. ${ }^{10,11}$ Noncognate help might also foster the activation of malignant IELs that arise in a subset of patients with refractory CD (reviewed in ref. 56). Indeed, these cells lack surface TCRs but contain granzyme B, express a large spectrum of NK receptors, and are highly sensitive to signals from IL$15{ }^{56}$ In keeping with this hypothesis, a gluten-free diet is indispensable even if not sufficient to improve symptoms in these patients. In summary, these data support the hypothesis that cooperation between gluten-specific DQ-restricted CD4 ${ }^{+}$ $\mathrm{T}$ cells and IL-15 is sufficient to orchestrate the activation of noncognate $\mathrm{CD}^{+}{ }^{+} \mathrm{T}$ cells and tissue damage in the intestine of CD patients (Figure 1). Whether such cooperation might participate in the onset of $\mathrm{CD}$-associated autoimmune diseases such as type 1 diabetes or thyroiditis is an interesting possibility but remains to be investigated. The mechanism(s) that stimulate(s) the excessive production of IL-15 by epithelial cells and of the $\alpha$ chain of its receptor in $\mathrm{CD}$ also remain(s) to be deciphered.

\section{Models investigating the role of SIgA in the permeability to dietary antigen}

The results described above highlight the remarkable resistance of mice to the induction of a celiac-like enteropathy. Interestingly, in the DQ8 model, a mild enteropathy was induced when mice were fed with gluten in the presence of cholera toxin that enhances intestinal permeability. ${ }^{29}$ It is also noticeable that feeding gluten triggered an enteropathy in $\mathrm{Rag}^{-1-}$ but not in nude mice transferred with gluten-specific T cells. ${ }^{10} \mathrm{~T}$ cell-transferred nude mice differ from $\mathrm{Rag}^{-1-}$ mice by their capacity to generate IgA against gluten. ${ }^{10}$ Human $\mathrm{CD}$ is associated with a strong anti-gliadin IgA response in the serum $^{64}$ and in the intestinal lumen. ${ }^{65}$ Yet, it is interesting that patients with selective IgA deficiency are more susceptible to $\mathrm{CD} .{ }^{66}$ Moreover, we have shown that, in active $\mathrm{CD}$, gliadinspecific SIgA1 lose their protective barrier function and rather behave as a Trojan horse because of their interactions with the CD71 receptor abnormally expressed at the apical enterocyte surface. ${ }^{65,67}$ Through their binding to CD71, SIgA1 complexed with gluten peptides are rapidly transported from the apical to the basal membrane via the recycling pathway, and immunostimulatory gluten peptides can be delivered into lamina propria. ${ }^{67}$ Mouse polymeric mouse IgA, in contrast to human SIgA1, does not bind CD71 and therefore cannot lose its protective role against inadvertent absorption of dietary antigens. In order to test the importance of this mechanism in promoting the transport of intact antigen and in activating 


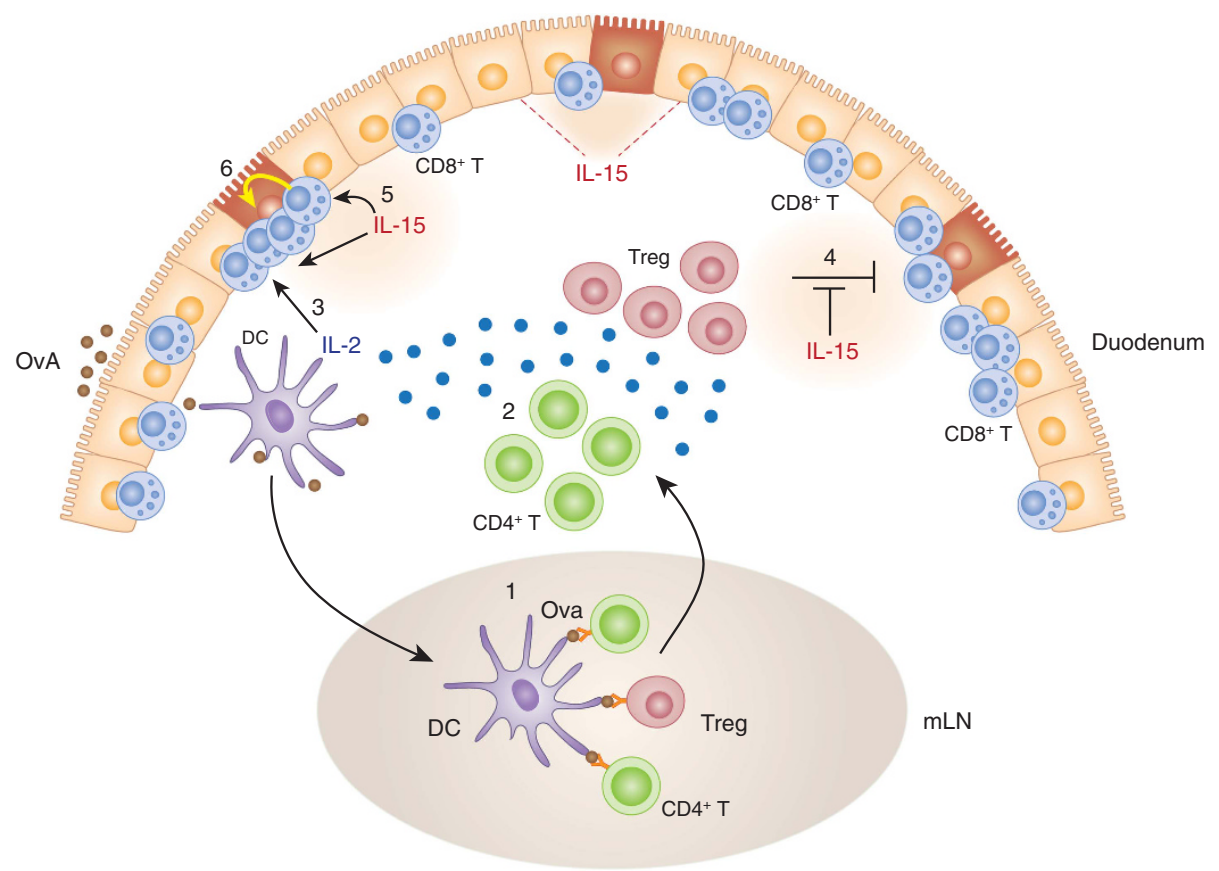

Figure 1 Activation of noncognate cytotoxic CD8 ${ }^{+}$T cells in celiac-like lesions of OTII/hIL-15Tge mice. Ovalbumin (OVA)-specific CD4 ${ }^{+}$T cells primed in Peyer's patches (not shown) or in mesenteric lymph nodes ( $\mathrm{mLNs}$ ) (1) home back into lamina propria, and secrete interleukin-2 (IL-2) upon restimulation by dietary OVA (2). IL-2 synergizes with epithelium-derived IL-15 to induce the expansion of resident effector CD8 ${ }^{+} \mathrm{T}$ cells and their acquisition of granzyme B (3). IL-15 prevents the inhibitory effect of FoxP3 ${ }^{+}$regulatory T cells (Tregs) on CD ${ }^{+} \mathrm{T}_{\text {cells }}(4)$ and stimulates their survival (5). As a consequence, cytotoxic $\mathrm{CD} 8^{+} \mathrm{T}$ cells expand in the intestinal tissue independently of the specificity of their T-cell receptor. Upon encounter with their cognate antigen in the tissue (which might be self or microbial antigens), $\mathrm{CD}^{+} \mathrm{T}$ cells can degranulate and cause tissue damage (6). Cooperation between IL-2 produced by CD4 ${ }^{+}$T cells specific for the dietary antigen and IL-15 might thus confer innate-like cytotoxic activity to resident intestinal effector/memory CD8 ${ }^{+}$T cells. DC, dendritic cell.

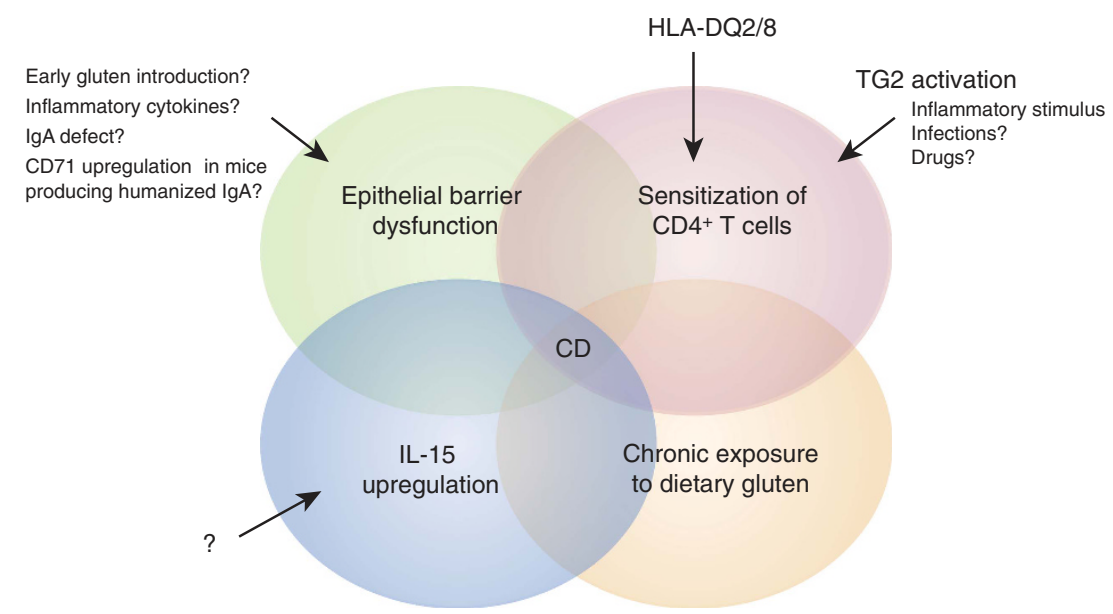

Figure 2 Multiple factors to combine for a mouse model of celiac-like enteropathy. No mouse model of celiac disease is yet available. The key putative factors predisposing to celiac disease (CD) that have been tested in rodent models are indicated in this scheme. Their combination is likely necessary to recapitulate CD but may be difficult to achieve. Better understanding of the mechanisms that control interleukin-15 (IL-15) upregulation and activation of transglutaminase 2 (TG2) is a necessary step. Whether CD71-SlgA interactions are important for epithelial dysfunction in CD also needs to be established. HLA, human leukocyte antigen; IgA, immunoglobulin A; SIgA, secretory IgA.

mucosal CD4 ${ }^{+}$T-cell responses, we designed a mouse model in which human IgA1s, which can bind to the mouse CD71 receptor, were provided by a hybridoma ${ }^{68}$ (Table $\mathbf{1}$ ). Hybridoma cells secreting OVA-specific monoclonal chimeric
hzIgA1 (made of human $\alpha 1$ heavy and of murine light chains) were grafted on the mouse back (Table 1). After 2 weeks, OVAspecific ShzIgA1s were detected in the intestinal lumen. Ectopic expression of CD71 was then induced at the apex of duodenal 
enterocytes by intraperitoneal administration of tyrphostin A8, a drug known to enhance CD71-mediated apical-to-basal transcytosis of insulin-transferrin complexes ${ }^{69,70}$ (Table 1). Increased transepithelial transport of intact OVA, but not of an irrelevant dietary antigen, could be demonstrated ex vivo using Ussing chambers. ${ }^{68}$ OVA-secreting hybridoma cells were next grafted onto transgenic Balb/c mice with OVA-specific transgenic $\mathrm{CD}^{+}{ }^{+} \mathrm{T}$ cells. Mice were treated with tyrphostin A 8 on days 13 and 14 and gavaged with OVA on days 14, 15, and 16 (Table 1). On day 20, a significant increase in the proportion of activated CD69 ${ }^{+}$OVA-specific $\mathrm{CD} 4{ }^{+} \mathrm{T}$ cells was observed in the mLNs of OVA-gavaged mice treated with tyrphostin A8. Moreover, mLN T cells secreted significantly more IFN $\gamma$ in response to OVA than T cells from control mice bearing an irrelevant hybridoma ${ }^{68}$ (Table 1). Altogether, these results support the hypothesis that SIgA-mediated transport of immunogenic gluten peptides may be one important step in the cascade of event that triggers a pathogenic immune response in CD. Recent evidence that binding of polymeric IgA to CD71 can trigger a signaling cascade and the production of inflammatory cytokines further sustains this hypothesis. ${ }^{71}$

\section{CONCLUSION}

Overall, the multiple attempts to develop a mouse model of CD have highlighted the robustness of the mechanisms that lead to and maintain tolerance to food antigens. These studies indicate that a quantitative increase in the frequency of gluten-specific $\mathrm{CD}^{+}{ }^{+} \mathrm{T}$ cells, as expected in individuals harboring at risk HLA-DQ molecules, is unlikely to be sufficient to trigger CD enteropathy, even if amplified upon activation of TG2 (Figure 2). Additional mechanisms are necessary to break the tolerance and to induce tissue damage (Figure 2). Among these mechanisms, IL-15 likely plays a central role (Figure 2). By cooperating with gluten-specific $\mathrm{CD} 4{ }^{+} \mathrm{T}$ cells and inhibiting immunoregulatory mechanisms, IL-15 can license the activation of noncognate cytotoxic effector lymphocytes. Prolonged exposure to dietary gluten seems however necessary to sustain the activation of CD $4{ }^{+}$T cells and to promote, in the presence of IL-15, sufficient accumulation of cytotoxic IEL to trigger tissue damage (Figure 2). In mouse models, anti-gliadin SIgA might exert a barrier effect protecting against pathologic sensitization by dietary gluten except at a very young age when the rodent intestinal barrier is highly permeable. In contrast, in humans, binding of SIgA1 to CD71 can circumvent the barrier effect of SIgA and promote mucosal entrance of immunogenic peptides (Figure 2). This effect, which depends on the upregulation of CD71, may occur at all ages notably as a consequence of reduced iron stocks. Altogether, these considerations underscore the wide spectrum of mechanisms that need to be combined to recapitulate human $\mathrm{CD}$ and explain the difficulties met to set up a pertinent model of CD that could be used to test innovative therapies (Figure 2). A better understanding of the mechanism(s) that lead(s) to chronic upregulation of IL-15 and to TG2 activation might help in the future to more accurately recapitulate $\mathrm{CD}$ in humanized mouse models. Delineating the exact role of CD71-SIgA interactions will also be necessary.

\section{ACKNOWLEDGMENTS}

The authors are supported by Institutional grants from Institut National Pour la Santé et la Recherche and Université Paris Descartes and by grants from la Ligue Contre le Cancer and la Fondation Princesse Grace. Institut Imagine is recipient of Investissement d'Avenir ANR-10-IAHU-01. Natalia Korneychuk was supported by a fellowship from the Ministère de l'Enseignement supérieur et de la Recherche and la Fondation pour la Recherche Médicale (code FDT20130927989).

\section{DISCLOSURE}

The authors declared no conflict of interest.

c 2015 Society for Mucosal Immunology

\section{REFERENCES}

1. Marietta, E.V. \& Murray, J.A. Animal models to study gluten sensitivity. Semin. Immunopathol. 34, 497-511 (2012).

2. Troncone, R. \& Ferguson, A. Animal model of gluten induced enteropathy in mice. Gut 32, 871-875 (1991).

3. Troncone, R., Caputo, N., Zibella, A., Molitierno, G., Maiuri, L. \& Auricchio, $\mathrm{S}$. Effects of gluten enriched diet on the small intestinal mucosa of normal mice and mice with graft versus host reaction. Gut 35, 779-782 (1994).

4. Troncone, R. \& Ferguson, A. Gliadin presented via the gut induces oral tolerance in mice. Clin. Exp. Immunol. 72, 284-287 (1988).

5. Stepankova, R., Tlaskalova-Hogenova, H., Sinkora, J., Jodl, J. \& Fric, P. Changes in jejunal mucosa after long-term feeding of germfree rats with gluten. Scand. J. Gastroenterol. 31, 551-557 (1996).

6. Stepankova, R., Kofronova, O., Tuckova, L., Kozakova, H., Cebra, J.J. \& Tlaskalova- Hogenova, H. Experimentally induced gluten enteropathy and protective effect of epidermal growth factor in artificially fed neonatal rats. J. Pediatr. Gastroenterol. Nutr. 36, 96-104 (2003).

7. Shan, L. etal. Structural basis for gluten intolerance in celiac sprue. Science 297, 2275-2279 (2002).

8. Abadie, V., Sollid, L.M., Barreiro, L.B. \& Jabri, B. Integration of genetic and immunological insights into a model of celiac disease pathogenesis. Annu. Rev. Immunol. 29, 493-525 (2011).

9. Meresse, B., Malamut, G. \& Cerf-Bensussan, N. Celiac disease: an immunological jigsaw. Immunity 36, 907-919 (2012).

10. Freitag, T.L. et al. Gliadin-primed CD4 + CD45RBlowCD25- T cells drive gluten-dependent small intestinal damage after adoptive transfer into lymphopenic mice. Gut 58, 1597-1605 (2009).

11. Freitag, T.L. et al. Testing safety of germinated rye sourdough in a celiac disease model based on the adoptive transfer of prolamin-primed memory T cells into lymphopenic mice. Am. J. Physiol. Gastrointest. Liver Physiol. 306, G526-G534 (2014).

12. Fuchs, A. et al. Intraepithelial type 1 innate lymphoid cells are a unique subset of IL-12- and IL-15-responsive IFN-gamma-producing cells. Immunity 38, 769-781 (2013).

13. Hmida, N.B. et al. Impaired control of effector T cells by regulatory T cells: a clue to loss of oral tolerance and autoimmunity in celiac disease?. Am. J. Gastroenterol. 107, 604-611 (2012).

14. Zanzi, D. et al. IL-15 interferes with suppressive activity of intestinal regulatory T cells expanded in Celiac disease. Am. J. Gastroenterol. 106, 1308-1317 (2011).

15. van Leeuwen, M.A. et al. Changes in natural Foxp3(+)Treg but not mucosally-imprinted CD62L(neg)CD38(+)Foxp3(+)Treg in the circulation of celiac disease patients. PLoS One 8, e68432 (2013).

16. Mottet, C., Uhlig, H.H. \& Powrie, F. Cutting edge: cure of colitis by CD4 + CD25 + regulatory T cells. J. Immunol. 170, 3939-3943 (2003).

17. Hammer, R.E., Maika, S.D., Richardson, J.A., Tang, J.P. \& Taurog, J.D. Spontaneous inflammatory disease in transgenic rats expressing HLA-B27 and human beta $2 \mathrm{~m}$ : an animal model of HLA-B27-associated human disorders. Cell 63, 1099-1112 (1990).

18. Nabozny, G.H. et al. HLA-DQ8 transgenic mice are highly susceptible to collagen-induced arthritis: a novel model for human polyarthritis. J. Exp. Med. 183, 27-37 (1996). 
19. Taneja, V. \& David, C.S. Role of HLA class II genes in susceptibility/ resistance to inflammatory arthritis: studies with humanized mice. Immunol. Rev. 233, 62-78 (2010).

20. Cosgrove, D. et al. Mice lacking MHC class II molecules. Cell 66, 10511066 (1991).

21. Black, K.E., Murray, J.A. \& David, C.S. HLA-DQ determines the response to exogenous wheat proteins: a model of gluten sensitivity in transgenic knockout mice. J. Immunol. 169, 5595-5600 (2002).

22. Law, Y.M., Yeung, R.S., Mamalaki, C., Kioussis, D., Mak, T.W. \& Flavell, R.A. Human CD4 restores normal Tcell development and function in mice deficient in murine CD4. J. Exp. Med. 179, 1233-1242 (1994).

23. van de Wal, Y. et al. Small intestinal T cells of celiac disease patients recognize a natural pepsin fragment of gliadin. Proc. Natl. Acad. Sci. USA 95, 10050-10054 (1998).

24. van de Wal, Y. et al. Selective deamidation by tissue transglutaminase strongly enhances gliadin-specific T cell reactivity. J. Immunol. 161, 15851588 (1998).

25. Cohn, A., Sofia, A.M. \& Kupfer, S.S. Type 1 diabetes and celiac disease: clinical overlap and new insights into disease pathogenesis. Curr. Diab. Rep. 14, 517 (2014)

26. Zhernakova, A., Withoff, S. \& Wijmenga, C. Clinical implications of shared genetics and pathogenesis in autoimmune diseases. Nat. Rev. Endocrinol. 9, 646-659 (2013).

27. Marietta, E. et al. A new model for dermatitis herpetiformis that uses HLA-DQ8 transgenic NOD mice. J. Clin. Invest. 114, 1090-1097 (2004).

28. Sardy, M., Karpati, S., Merkl, B., Paulsson, M. \& Smyth, N. Epidermal transglutaminase (TGase 3 ) is the autoantigen of dermatitis herpetiformis. J. Exp. Med. 195, 747-757 (2002).

29. Galipeau, H.J. et al. Sensitization to gliadin induces moderate enteropathy and insulitis in nonobese diabetic-DQ8 mice. J. Immunol. 187, 4338-4346 (2011).

30. Hansen, C.H. et al. A maternal gluten-free diet reduces inflammation and diabetes incidence in the offspring of NOD mice. Diabetes 63, 2821-2832 (2014).

31. Marietta, E.V. et al. Low incidence of spontaneous type 1 diabetes in nonobese diabetic mice raised on gluten-free diets is associated with changes in the intestinal microbiome. PLoS One 8, e78687 (2013).

32. Jaakkola, I., Jalkanen, S. \& Hanninen, A. Diabetogenic T cells are primed both in pancreatic and gut-associated lymph nodes in NOD mice. Eur. J. Immunol. 33, 3255-3264 (2003).

33. Yang, X.D., Sytwu, H.K., McDevitt, H.O. \& Michie, S.A. Involvement of beta 7 integrin and mucosal addressin cell adhesion molecule-1 (MAdCAM-1) in the development of diabetes in obese diabetic mice. Diabetes 46, 15421547 (1997).

34. Hanninen, A., Jaakkola, I. \& Jalkanen, S. Mucosal addressin is required for the development of diabetes in nonobese diabetic mice. J. Immunol. 160 6018-6025 (1998).

35. de Kauwe, A.L. et al. Resistance to celiac disease in humanized HLA-DR3DQ2-transgenic mice expressing specific anti-gliadin CD4 + T cells. J. Immunol. 182, 7440-7450 (2009).

36. Bodd, M., Raki, M., Bergseng, E., Jahnsen, J., Lundin, K.E. \& Sollid, L.M. Direct cloning and tetramer staining to measure the frequency of intestinal gluten-reactive T cells in celiac disease. Eur. J. Immunol. 43, 2605-2612 (2013).

37. Du Pre, M.F. et al. Tolerance to ingested deamidated gliadin in mice is maintained by splenic, type 1 regulatory T cells. Gastroenterology 141 610-620. 20 e1-2 (2011).

38. Sjostrom, $\mathrm{H}$. et al. Identification of a gliadin T-cell epitope in coeliac disease: general importance of gliadin deamidation for intestinal T-cell recognition. Scand. J. Immunol. 48, 111-115 (1998).

39. Hadis, U. et al. Intestinal tolerance requires gut homing and expansion of FoxP3 + regulatory Tcells in the lamina propria. Immunity 34, 237-246 (2011)

40. Abadie, V. \& Jabri, B. IL-15: a central regulator of celiac disease immunopathology. Immunol. Rev. 260, 221-234 (2014).

41. Di Sabatino, A. et al. Epithelium derived interleukin 15 regulates intraepithelial lymphocyte Th1 cytokine production, cytotoxicity, and survival in coeliac disease. Gut 55, 469-477 (2006).

42. Mention, J.J. et al. Interleukin 15: a key to disrupted intraepithelial lymphocyte homeostasis and lymphomagenesis in celiac disease. Gastroenterology 125, 730-745 (2003).
43. Bernardo, D. et al. Higher constitutive IL15R alpha expression and lower IL15 response threshold in coeliac disease patients. Clin. Exp. Immunol. 154, 64-73 (2008).

44. Hue, S. et al. A direct role for NKG2D/MICA interaction in villous atrophy during celiac disease. Immunity 21, 367-377 (2004).

45. Meresse, B. et al. Coordinated induction by IL15 of a TCR-independent NKG2D signaling pathway converts CTL into lymphokine-activated killer cells in celiac disease. Immunity 21, 357-366 (2004).

46. Malamut, G. et al. IL-15 triggers an antiapoptotic pathway in human intraepithelial lymphocytes that is a potential new target in celiac diseaseassociated inflammation and lymphomagenesis. J. Clin. Invest. 120, 2131-2143 (2010)

47. Ben Ahmed, M. et al. IL-15 renders conventional lymphocytes resistant to suppressive functions of regulatory $T$ cells through activation of the phosphatidylinositol 3-kinase pathway. J. Immunol. 182, 6763-6770 (2009).

48. Benahmed, M. et al. Inhibition of TGF-beta signaling by IL-15: a new role for $\mathrm{IL}-15$ in the loss of immune homeostasis in celiac disease. Gastroenterology 132, 994-1008 (2007).

49. Fehniger, T.A. et al. Fatal leukemia in interleukin 15 transgenic mice follows early expansions in natural killer and memory phenotype CD8 + Tcells. J. Exp. Med. 193, 219-231 (2001).

50. DePaolo, R.W. et al. Co-adjuvant effects of retinoic acid and IL-15 induce inflammatory immunity to dietary antigens. Nature 471, 220-224 (2011).

51. Ohta, N. et al. IL-15-dependent activation-induced cell death-resistant Th1 type CD8 alpha beta +NK1.1 + T cells for the development of small intestinal inflammation. J. Immunol. 169, 460-468 (2002).

52. Korneychuk, N. et al. Interleukin 15 and CD4(+) T cells cooperate to promote small intestinal enteropathy in response to dietary antigen. Gastroenterology 146, 1017-1027 (2014).

53. Yokoyama, S., Takada, K., Hirasawa, M., Perera, L.P. \& Hiroi, T. Transgenic mice that overexpress human IL-15 in enterocytes recapitulate both $B$ and T cell-mediated pathologic manifestations of celiac disease. J. Clin. Immunol. 31, 1038-1044 (2011).

54. Yokoyama, S., Perera, P.Y., Waldmann, T.A., Hiroi, T. \& LP., Perera Tofacitinib, a janus kinase inhibitor demonstrates efficacy in an IL-15 transgenic mouse model that recapitulates pathologic manifestations of celiac disease. J. Clin. Immunol. 33, 586-594 (2013).

55. Yokoyama, S. et al. Antibody-mediated blockade of IL-15 reverses the autoimmune intestinal damage in transgenic mice that overexpress IL-15 in enterocytes. Proc. Natl. Acad. Sci. USA 106, 15849-15854 (2009).

56. Malamut, G., Meresse, B., Cellier, C. \& Cerf-Bensussan, N. Refractory celiac disease: from bench to bedside. Semin. Immunopathol. 34, 601613 (2012)

57. Barnden, M.J., Allison, J., Heath, W.R. \& Carbone, F.R. Defective TCR expression in transgenic mice constructed using CDNA-based alpha- and beta-chain genes under the control of heterologous regulatory elements. Immunol. Cell Biol. 76, 34-40 (1998).

58. Newberry, R.D., Stenson, W.F. \& Lorenz, R.G. Cyclooxygenase-2dependent arachidonic acid metabolites are essential modulators of the intestinal immune response to dietary antigen. Nat. Med. 5, 900-906 (1999).

59. Fina, D. et al. Interleukin 21 contributes to the mucosal T helper cell type 1 response in coeliac disease. Gut 57, 887-892 (2008).

60. Bodd, M. et al. HLA-DQ2-restricted gluten-reactive T cells produce IL-21 but not IL-17 or IL-22. Mucosal Immunol. 3, 594-601 (2010).

61. Sarra, M. et al. IL-15 positively regulates IL-21 production in celiac disease mucosa. Mucosal Immunol. 6, 244-255 (2013).

62. Meresse, B. et al. Reprogramming of CTLs into natural killer-like cells in celiac disease. J. Exp. Med. 203, 1343-1355 (2006).

63. Cerwenka, A. et al. Retinoic acid early inducible genes define a ligand family for the activating NKG2D receptor in mice. Immunity 12, 721-727 (2000).

64. Giersiepen, K. et al. Accuracy of diagnostic antibody tests for coeliac disease in children: summary of an evidence report. J. Pediatr. Gastroenterol. Nutr. 54, 229-241 (2012).

65. Matysiak-Budnik, T. et al. Secretory lgA mediates retrotranscytosis of intact gliadin peptides via the transferrin receptor in celiac disease. J. Exp. Med. 205, 143-154 (2008)

66. Dominguez, O., Giner, M.T., Alsina, L., Martin, M.A., Lozano, J. \& Plaza, A.M. Clinical phenotypes associated with selective IgA deficiency: a review 


\section{REVIEW}

of 330 cases and a proposed follow-up protocol. An. Pediatr. 76, 261-267 (2012).

67. Lebreton, C. et al. Interactions among secretory immunoglobulin A, CD71, and transglutaminase-2 affect permeability of intestinal epithelial cells to gliadin peptides. Gastroenterology 143, 698-707. e1-e4 (2012).

68. Abed, J. et al. Abnormal apical-to-basal transport of dietary ovalbumin by secretory IgA stimulates a mucosal Th1 response. Mucosal Immunol. 7, 315-324 (2014).

69. Norouziyan, F., Shen, W.C. \& Hamm-Alvarez, S.F. Tyrphostin A8 stimulates a novel trafficking pathway of apically endocytosed transferrin through Rab11-enriched compartments in Caco-2 cells. Am. J. Physiol. Cell Physiol. 294, C7-21 (2008).

70. Xia, C.Q. \& Shen, W.C. Tyrphostin-8 enhances transferrin receptormediated transcytosis in Caco-2- cells and inreases hypoglycemic effect of orally administered insulin-transferrin conjugate in diabetic rats. Pharm. Res. 18, 191-195 (2001).

71. Tamouza, $\mathrm{H}$. et al. The IgA1 immune complex-mediated activation of the MAPK/ERK kinase pathway in mesangial cells is associated with glomerular damage in IgA nephropathy. Kidney Int. 82, 1284-1296 (2012).

72. Wieser, H. Chemistry of gluten proteins. Food Microbiol. 24, 115-119 (2007).

73. Sollid, L.M., Qiao, S.W., Anderson, R.P., Gianfrani, C. \& Koning, F. Nomenclature and listing of celiac disease relevant gluten T-cell epitopes restricted by HLA-DQ molecules. Immunogenetics 64, 455-460 (2012).

74. Junker, Y. et al. Wheat amylase trypsin inhibitors drive intestinal inflammation via activation of toll-like receptor 4. J. Exp. Med. 209, 2395-2408 (2012). 